\title{
Metaproteomics reveals growth phase-dependent responses of an in vitro gut microbiota to metformin
}

Zikai Hao ${ }^{1,2,3}$, Leyuan $\mathrm{Li}^{2}$, Zhibin $\mathrm{Ning}^{2}, \mathrm{Xu}$ Zhang ${ }^{2}$, Janice Mayne ${ }^{2}$, Kai Cheng ${ }^{2}$, Krystal Walker $^{2}$, Hong Liu ${ }^{1,3_{*}}$, Daniel Figeys ${ }^{1,2_{* *}}$

${ }^{1}$ Beijing Advanced Innovation Centre for Biomedical Engineering, Beihang University, Beijing, China

${ }^{2}$ Department of Biochemistry, Microbiology and Immunology, Ottawa Institute of Systems Biology, Faculty of Medicine, University of Ottawa, Ottawa, Canada.

${ }^{3}$ School of Biological Science and Medical Engineering, Beihang University, Beijing, China

* Correspondence to Hong Liu, e-mail: $\underline{1 \text { h64@ @uaa.edu.cn }}$

**Correspondence to Daniel Figeys, e-mail: dfigeys@ uottawa.ca 
Supplementary Figure 1

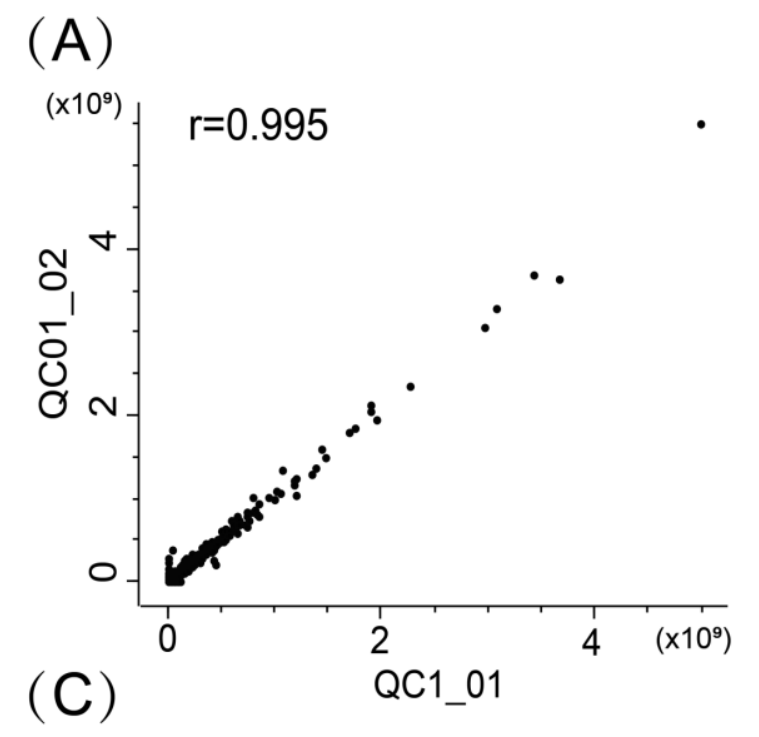

(B)
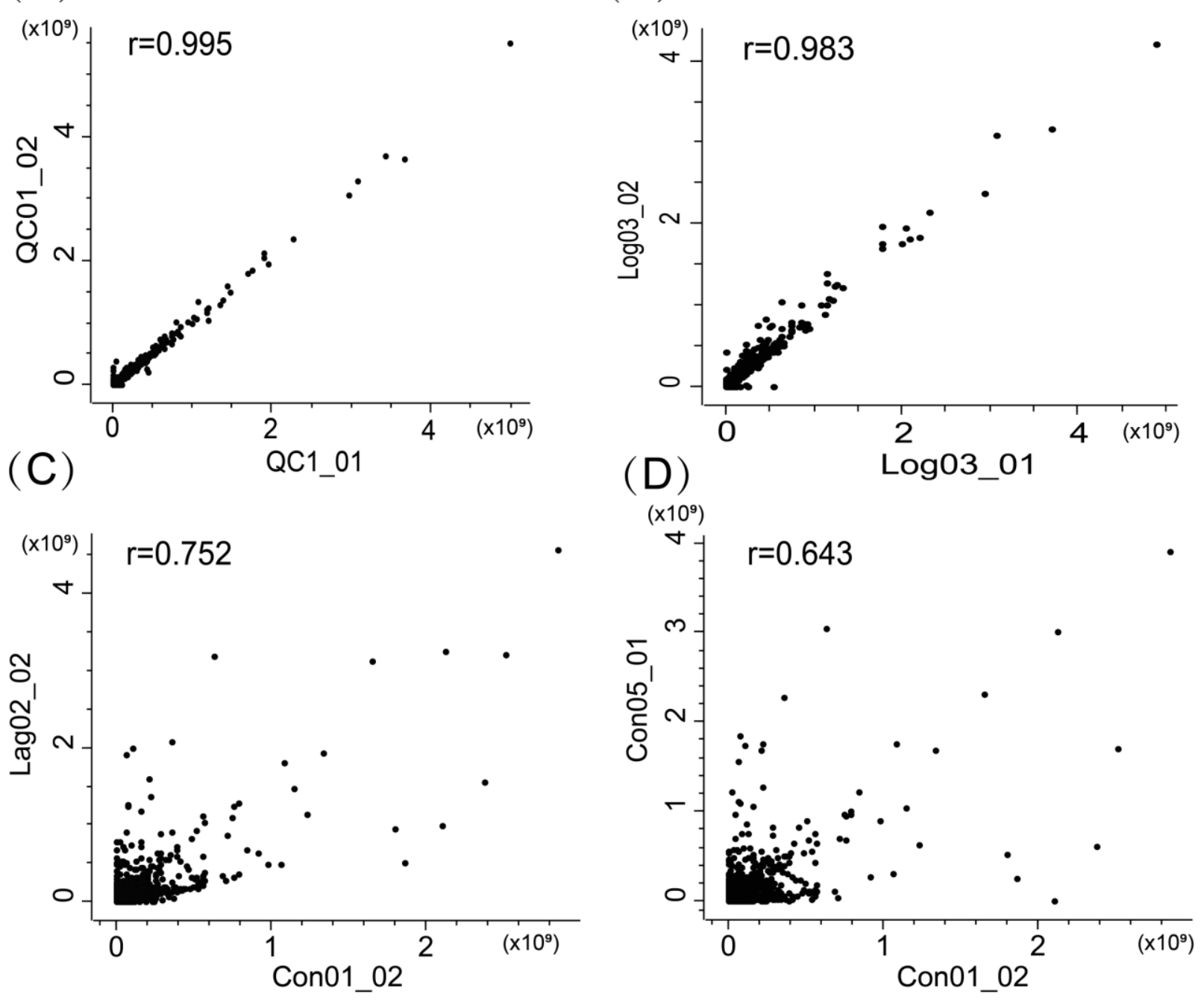

Figure S1. Evaluation of data quality. Both QC (A) and technical replicates (B) showed high degrees of correlation ( $r$ value), while changes among treatment (C) and changes over time (D) were observed. Scatter plots were plotted with transformed LFQ protein intensity. Pearson's correlation coefficient $r$ is shown. 

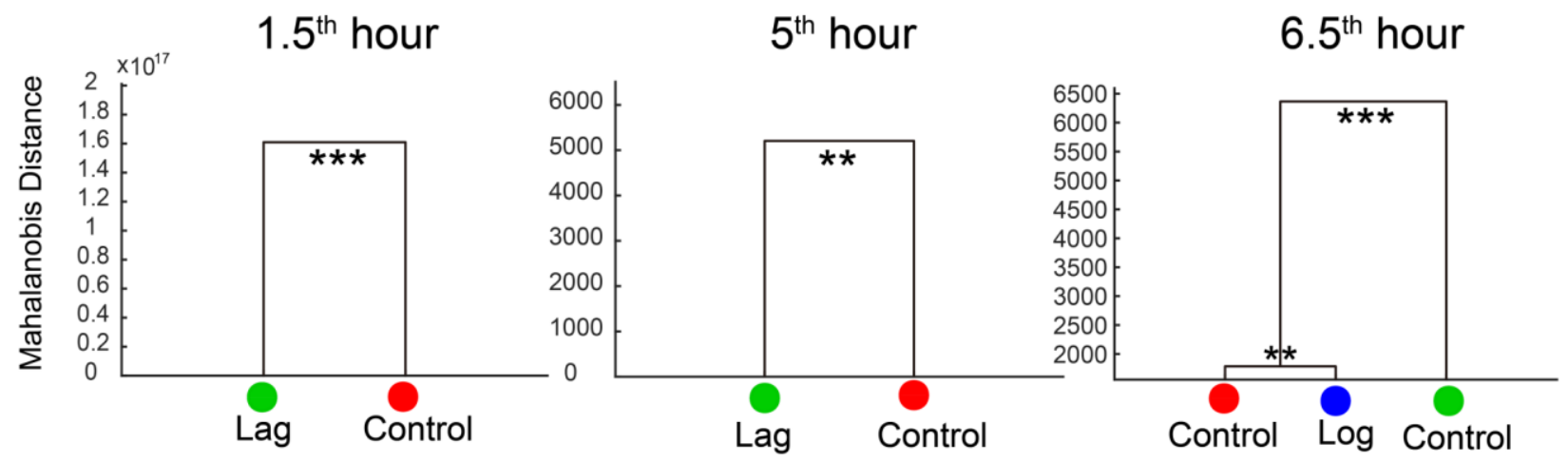

$12^{\text {th }}$ hour
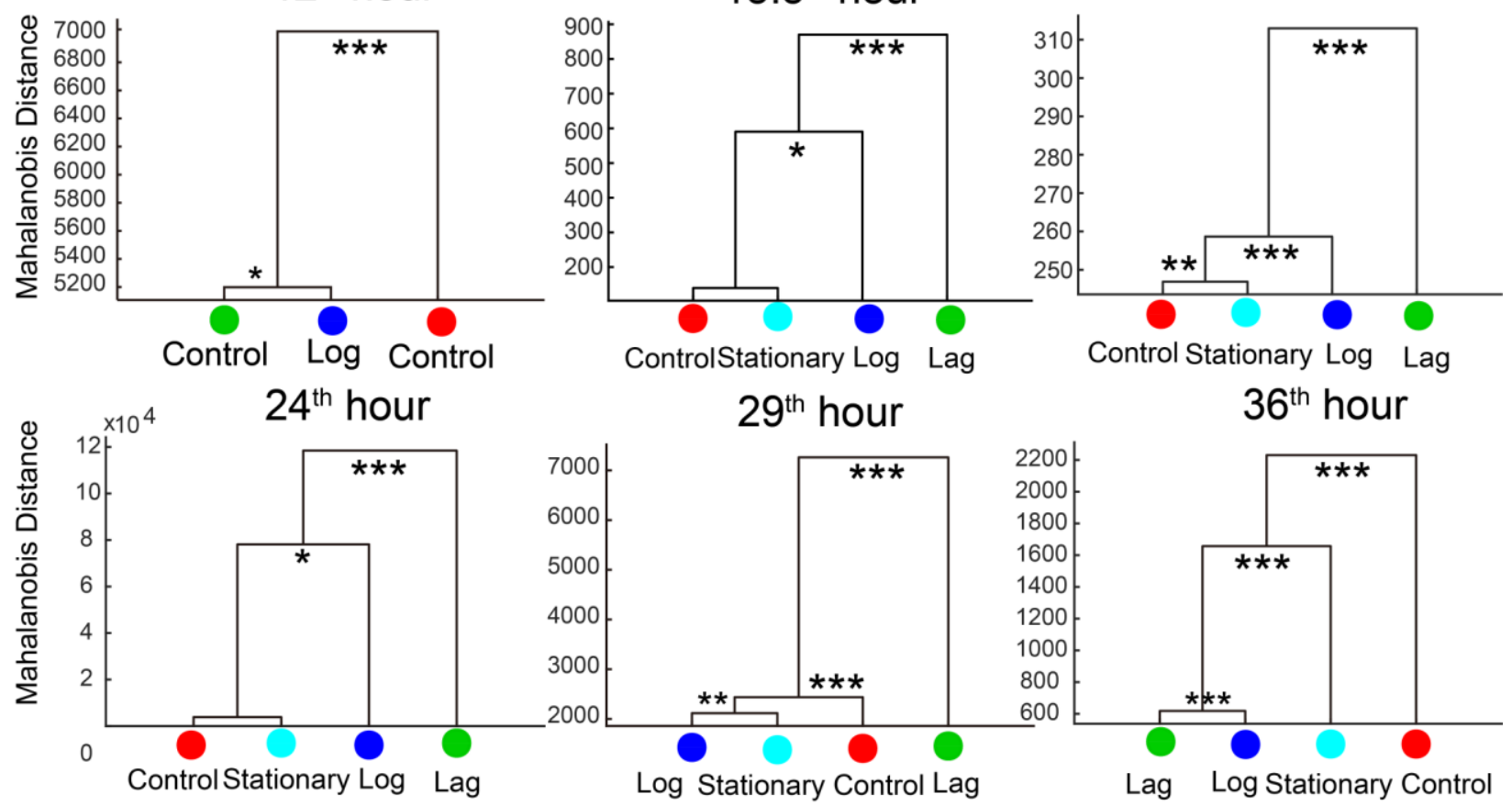

Figure S2. MANOVA analysis of the different groups over time. The statistical significance of the separation among different groups was assessed by MANOVA test based on Mahalanobis distances using the first 20 PCs of PCA at the $1.5^{\text {th }}, 5^{\text {th }}, 6.5^{\text {th }}, 12^{\text {th }}, 13.5^{\text {th }}, 17^{\text {th }}, 24^{\text {th }}, 29^{\text {th }}$ and $36^{\text {th }}$ hour. The clusters are computed by applying the single linkage method to the matrix of Mahalanobis distances between group means. ${ }^{*} \mathrm{p}<0.05,{ }^{*} \mathrm{p}<0.01, * * * \mathrm{p}<0.001$. 


\section{Supplementary Figure 3}

(A) Change in relative abundance of microbiome genus
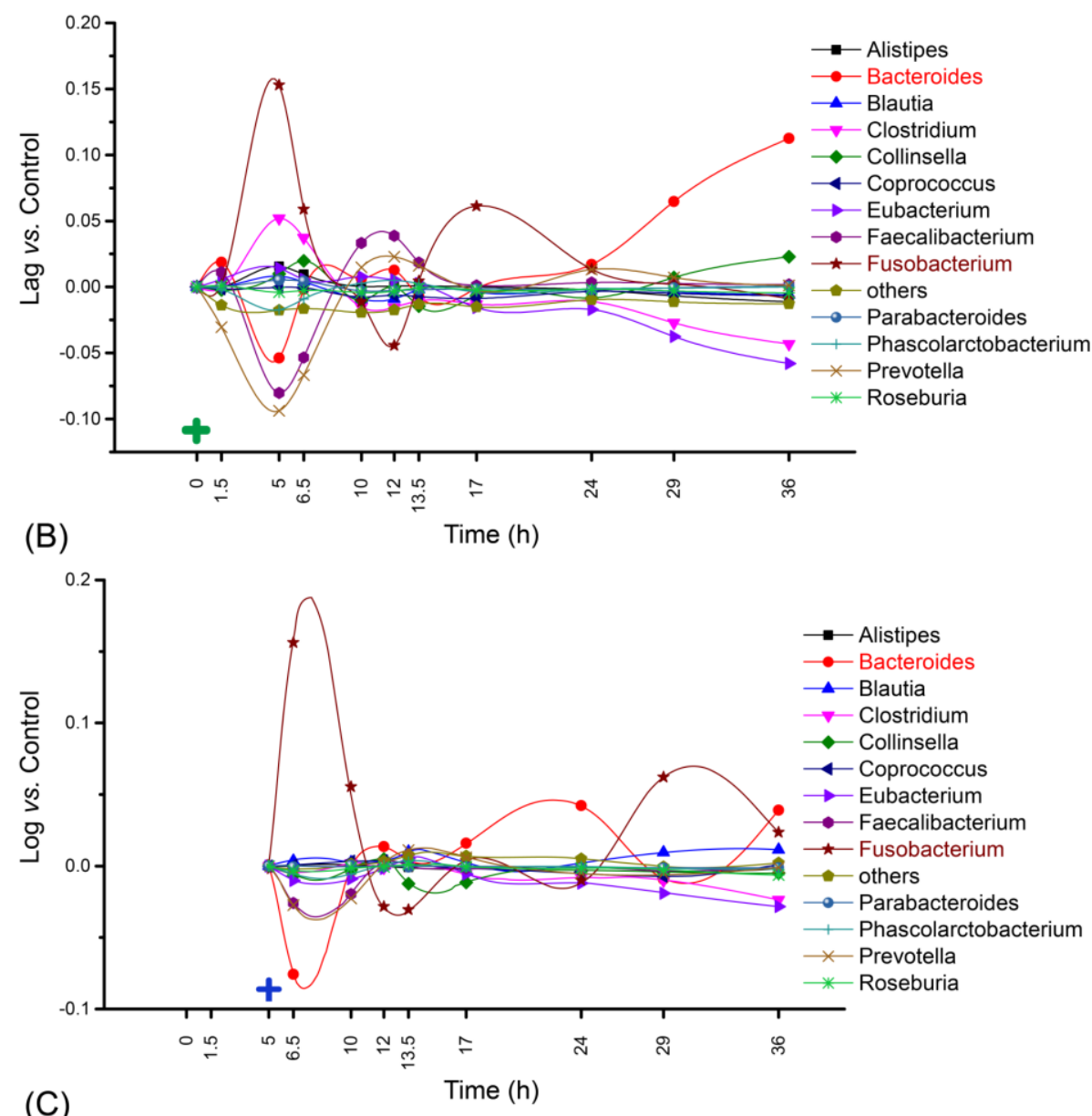

$(\mathrm{C})$

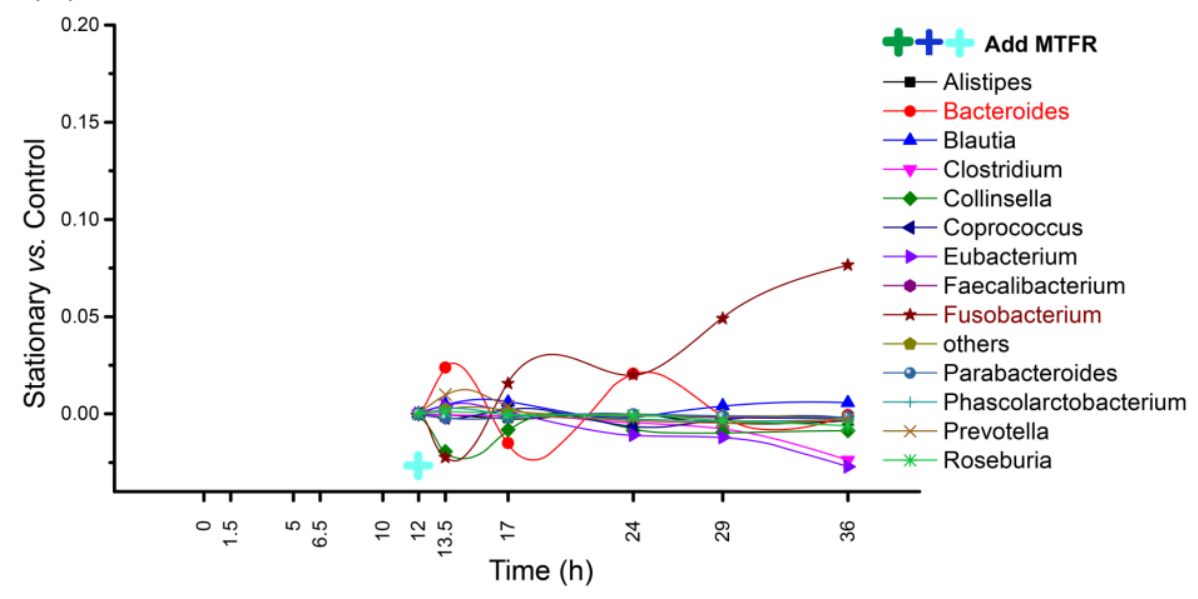

Figure S3. Change in relative abundance of the microbiome genera between the treatment group and control group over time. A, Lag vs. Control; B, Log vs. Control; C, vs. Control. 


\section{Supplementary Figure 4}

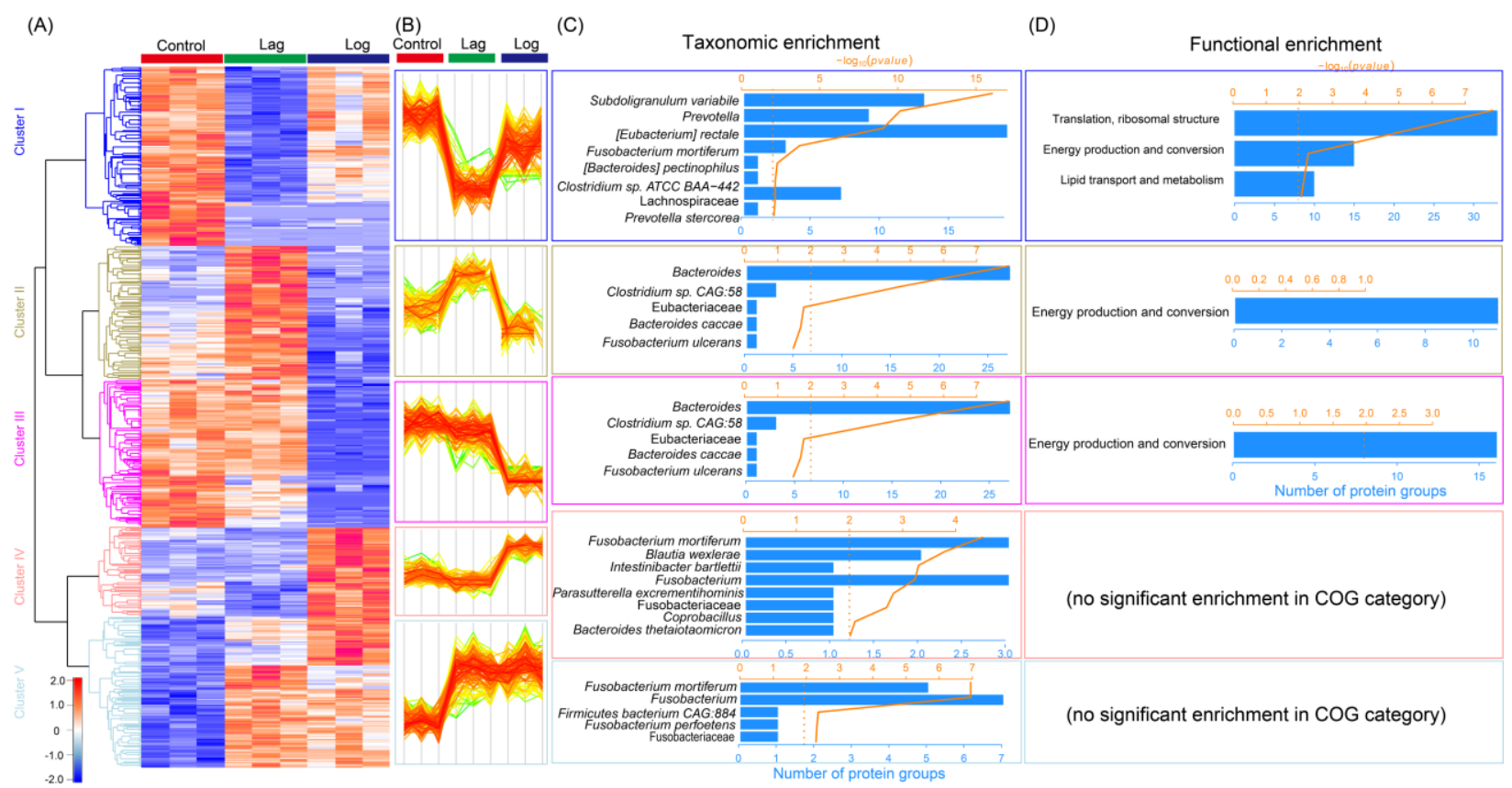

Figure S4. Metaproteomics revealed alterations of taxonomic \& functional composition among the different treatment groups at $6.5^{\text {th }}$ hour. (A) Heat map of significantly altered (FDR-adjusted $\mathrm{p}<0.05$, one-way ANOVA with Fisher's LSD test) protein groups among different groups at $6.5^{\text {th }}$ hour. Heatmap shows the LFQ intensity of altered protein groups for each sample, 5 clusters (painted in different colors) containing >10 proteins were divided for further analyzed. (B) Alterations of the LFQ intensity of protein groups in each cluster. (C, D) Alterations of taxonomic \& functional composition in each cluster. All the significantly altered taxonomy with a p-value cut off of 0.01 and the significantly altered COGs with a p-value cut off of 0.05 are shown. Bar charts showed the total numbers of protein groups corresponding to each taxonomy or COG categories (FDR-adjusted $\mathrm{P}<0.05$ ). 


\section{Supplementary Figure 5}

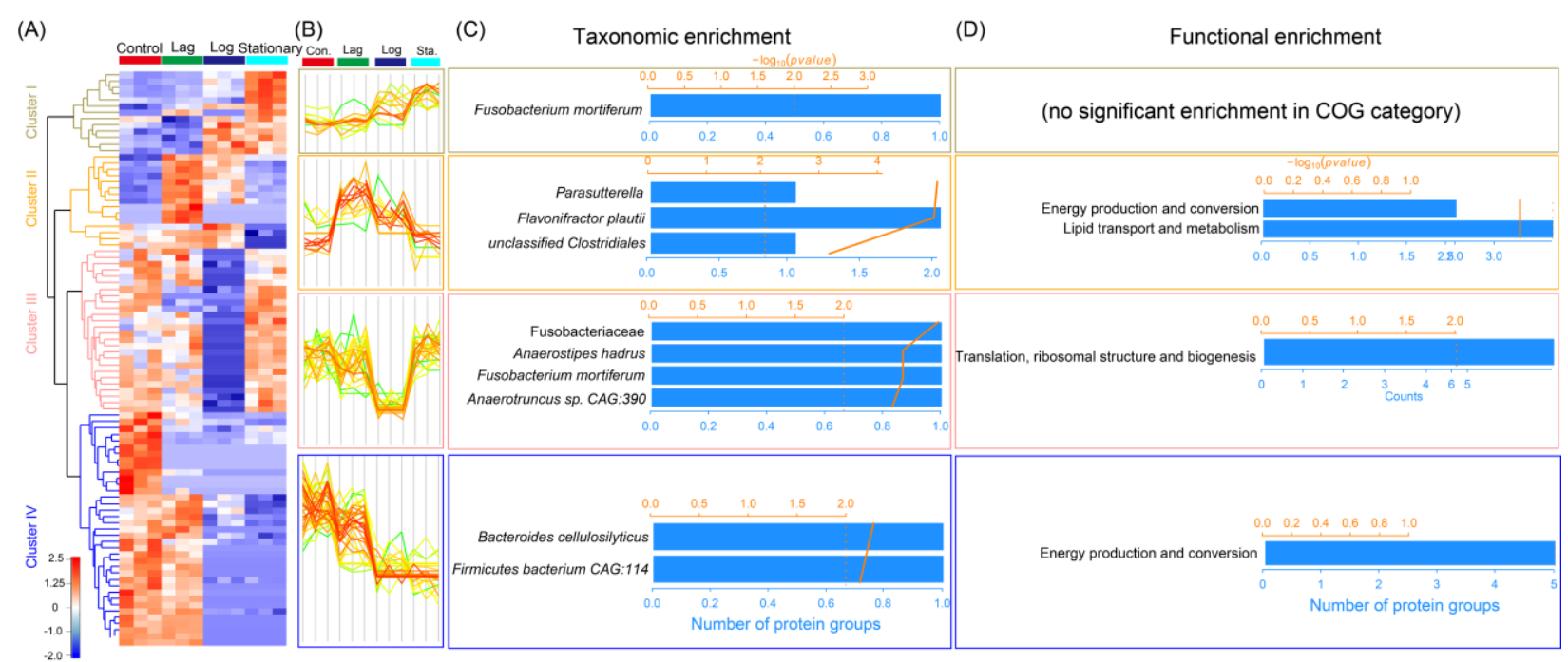

Figure S5. Metaproteomics revealed alterations of taxonomic \& functional composition among the different treatment groups at $17^{\text {th }}$ hour. (A) Heat map of significantly altered (FDR-adjusted $\mathrm{p}<0.05$, one-way ANOVA with Fisher's LSD test) protein groups among different groups at $17^{\text {th }}$ hour. Heatmap shows the LFQ intensity of altered protein groups for each sample, 4 clusters (painted in different colors) containing >10 proteins were divided for further analyzed. (B) Alterations of the LFQ intensity of protein groups in each cluster. (C, D) Alterations of taxonomic \& functional composition in each cluster. All the significantly altered taxonomy with a p-value cut off of 0.01 and all the significantly altered COGs with a p-value cut off of 0.05 are shown. Bar charts showed the total numbers of protein groups corresponding to each taxonomy or COG categories (FDR-adjusted $\mathrm{P}<0.05$ ). 


\section{Supplementary Figure 6}

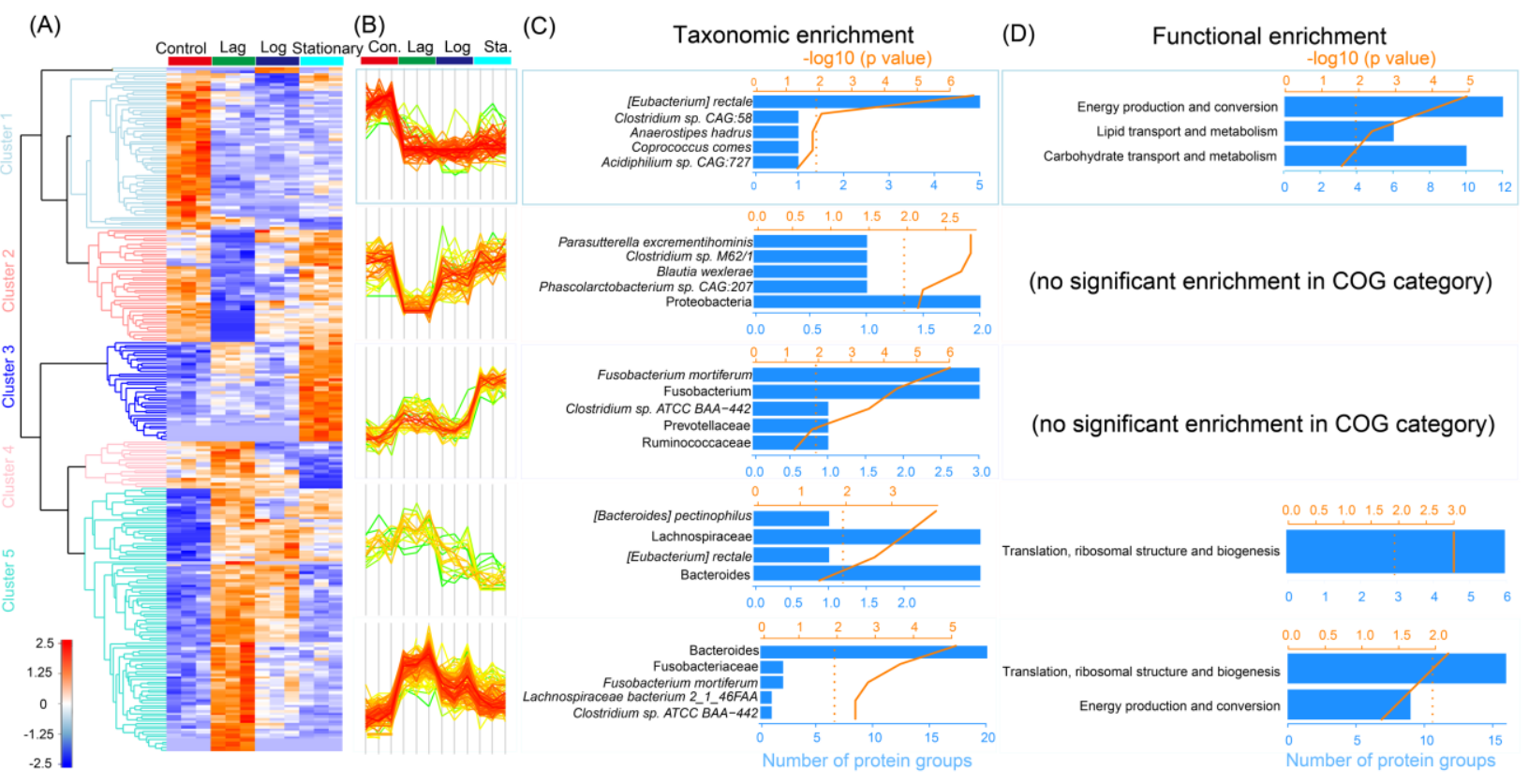

Figure S6. Metaproteomics revealed alterations of taxonomic \& functional composition among the different treatment groups at $36^{\text {th }}$ hour. (A) Heat map of significantly altered (FDR-adjusted $p<0.05$, one-way ANOVA with Fisher's LSD test) protein groups among different groups at $36^{\text {th }}$ hour. Heatmap shows the LFQ intensity of altered protein groups for each sample, 5 clusters (painted in different colors) containing $>10$ proteins were divided for further analyzed. (B) Alterations of protein groups in each cluster. (C, D) Alterations of taxonomic \& functional composition in each cluster. Top 5 most significant altered taxonomy and all the significantly altered COGs with a p-value cut off of 0.05 are shown. Bar charts showed the total numbers of protein groups corresponding to each taxonomy or COG categories (FDR-adjusted $\mathrm{P}<0.05$ ). 


\section{Supplementary Figure 7}

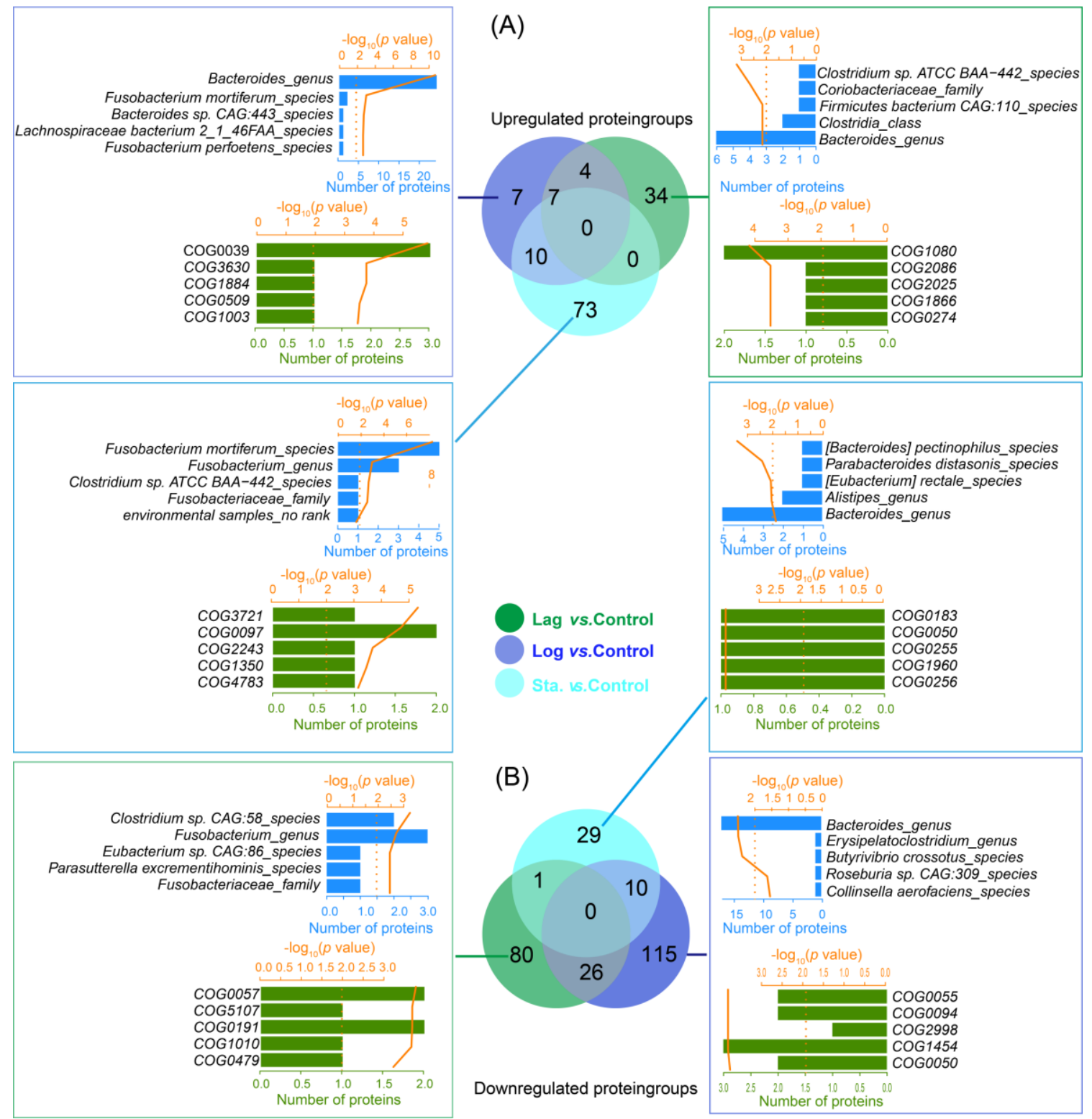

Figure S7. The alterations of taxonomic \& functional composition among the different treatment groups after $12 \mathrm{hr}$ of stimulation. Numbers of the significantly upregulated (A) and downregulated (B) protein groups in treatment groups compared to controls (FDR-adjusted $\mathrm{p}<0.05, t$-test). Venn diagram indicates that the most of significantly altered protein groups in the different treatment group were unique. The unique protein groups in each treatment group was used for taxonomic \& functional enrichment analysis. Top 5 most significant altered taxonomy and COGs with a p-value cut off of 0.05 are shown. Bar charts showed the total numbers of protein groups corresponding to each taxonomy or COG categories (FDR-adjusted $\mathrm{P}<0.05$ ). 


\section{Supplementary Figure 8}

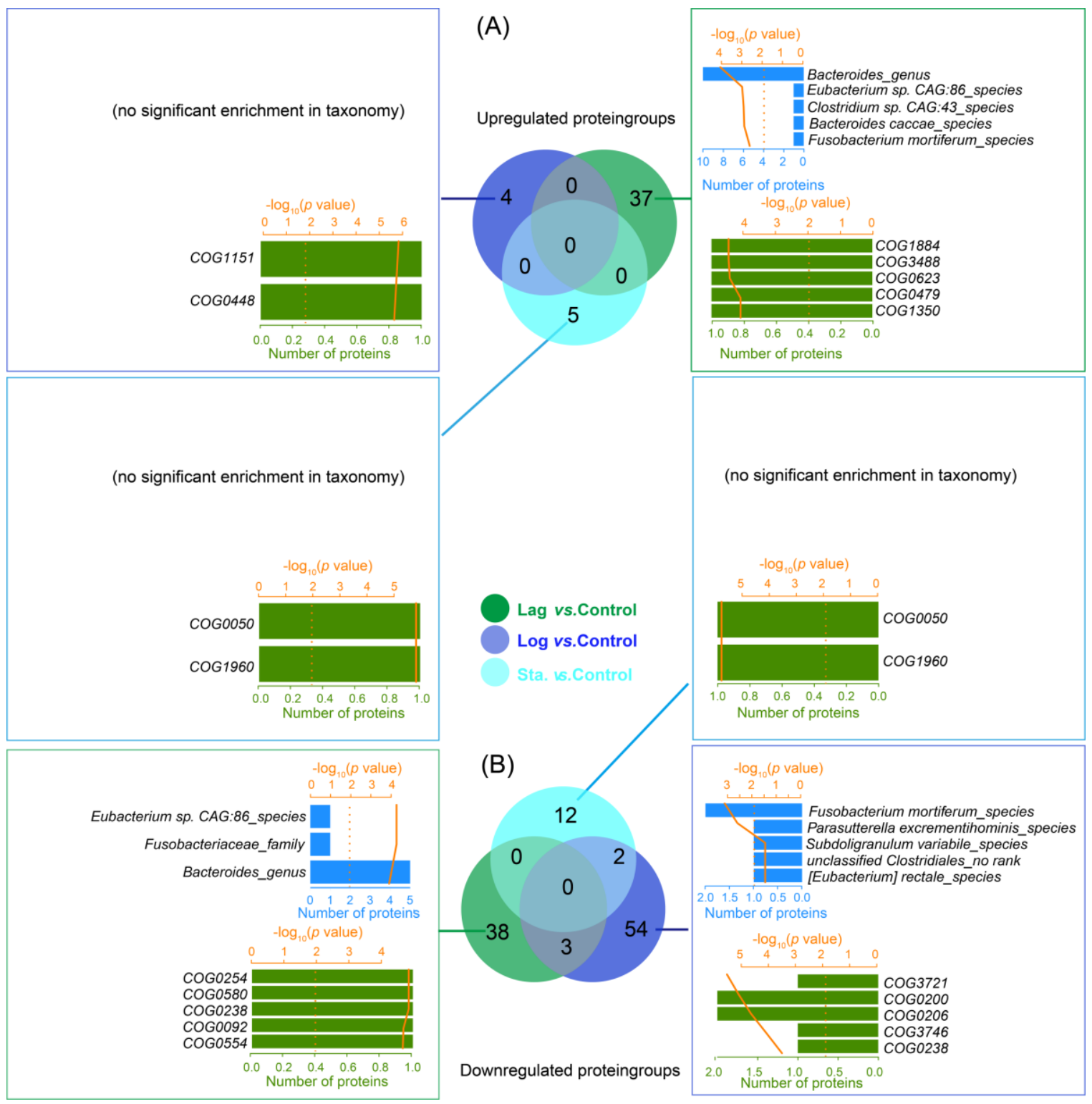

Figure S8. The alterations of taxonomic \& functional composition among the different treatment groups after $24 \mathrm{hr}$ of stimulation. Numbers of the significantly upregulated (A) and downregulated (B) protein groups in treatment groups compared to controls (FDR-adjusted $\mathrm{p}<0.05, t$-test). Venn diagram indicates that the most of significantly altered protein groups in the different treatment group were unique. The unique protein groups in each treatment group was used for taxonomic \& functional enrichment analysis. Top 5 most significant altered taxonomy and COGs with a p-value cut off of 0.05 are shown. Bar charts showed the total numbers of protein groups corresponding to each taxonomy or COG categories (FDR-adjusted $\mathrm{P}<0.05$ ). 